\title{
Global tracking and state estimation with nonsmooth impacts for a mass confined to an $n$-dimensional half-space ${ }^{\star}$
}

\author{
Fulvio Forni ${ }^{*}$ Andrew R. Teel ${ }^{* *}$ Luca Zaccarian ${ }^{* * *}$ \\ * Department of Electrical Engineering and Computer Science, \\ University of Liège, 4000 Liège, Belgium (e-mail: fforni@ulg.ac.be) \\ ** Dept. of Electrical and Computer Engineering, Univ. of California, \\ Santa Barbara, CA 93106, USA (e-mail: teel@ece.ucsb.edu) \\ *** CNRS; LAAS; 7 av. du Colonel Roche F-31077, Toulouse, France. \\ Universit de Toulouse; UPS, INSA, INP, ISAE, UT1, UTM, LAAS; \\ F-31077, Toulouse, France (e-mail: zaccarian@laas.fr)
}

\begin{abstract}
We formulate tracking and state-estimation problems of a translating mass moving in an $n$-dimensional space and impacting on a hyperplane. Due to the discontinuous trajectories arising from the nonsmooth impacts, we use hybrid systems stability analysis tools to establish that 1) a tracking control algorithm and 2) an observer algorithm guarantee global exponential stability. Then, based on a separation principle, we combine the two constructions above to design a dynamic output feedback controller ensuring asymptotic tracking.
\end{abstract}

Keywords: hybrid systems, nonsmooth impacts, hybrid tracking, hybrid observer

\section{INTRODUCTION}

Mechanical systems with dynamics subject to nonsmooth impacts have been subject of extensive investigations in the past two decades. In particular, several Lyapunovbased solutions to their stabilization and tracking problem have been proposed in Brogliato (2004); Leine and van de Wouw (2008); Tornambe (1999), and several studies have been developed for the dual state-estimation problem Menini and Tornambè (2001b,a); Galeani et al. (2003). Some of them address the problem via the larger class of complementarity Lagrangian systems. These systems are a specific class of hybrid systems where the state is subject to a jump or re-initialization rule whenever a unilateral constraint is reached (see Heemels and Brogliato (2003) for a survey and Morarescu and Brogliato (2010) for a recent work which gives an updated overview of the results in this field). The parallel problem of tracking trajectories while restricting the control action at the impact times is addressed in Sanfelice et al. (2007b) and references therein.

Recently, Forni et al. (2011b,c) proposed a novel view of the tracking problem for impacting systems constrained to evolve in a planar region delimited by suitable edges corresponding to lines Forni et al. (2011b) or curves whose gradient satisfies a Lipschitz condition Forni et al. (2011c). The novelty of the new approach therein proposed is twofold: it relies on the hybrid systems framework of

\footnotetext{
* This paper presents research results of the Belgian Network DYSCO (Dynamical Systems, Control, and Optimization), funded by the Interuniversity Attraction Poles Programme, initiated by the Belgian State, Science Policy Office. The scientific responsibility rests with its authors. Research supported in part by AFOSR grant FA9550-09-1-0203 and NSF grants ECCS-0925637 and CNS0720842 .
}

Goebel et al. (2012) (see also Goebel et al. (2009)) and it uses a novel concept of "mirrored reference", which is exploited in such a way that the tracking system decides what target (reference) should be tracked based on the minimum of several Lyapunov functions. Later, in Forni et al. (2011a), this idea was further developed in such a way to exploit the recent results of Teel et al. (2011) to assess exponential stability of the error dynamics of the tracking scheme, in addition to extending the results to the dual state estimation problem and combining the two schemes using a separation principle. Moreover, in Forni et al. (2011a) the "min" rule introduced in Forni et al. (2011b,c) was efficiently replaced by a suitable update law defined by an automaton. With the above extensions, the local asymptotic stability results of Forni et al. (2011b,c) was extended in Forni et al. (2011a) to global exponential stability.

In this paper, we further develop on the results discussed above by illustrating the generalization of the corresponding constructions to an $n$-dimensional state-space. To keep the presentation simple, we only focus on a hyperplane dividing the $n$-dimensional space in two half-spaces. For this special case, many of the technical assumptions required in Forni et al. (2011a,c) are automatically satisfied so that our derivations are simplified. In particular, in Section 2 we introduce the setup data and some preliminary results. In Sections 3 and 4 we address and solve the tracking and observation problems, respectively. Finally, in Section 5 we combine the two results above to achieve dynamic output feedback tracking.

Notation: The Euclidean norm of a vector is denoted by $|\cdot|$. For any given vectors $v$ and $w,\langle v, w\rangle=v^{T} w$. For $n \in \mathbb{N}, I_{n}$ denotes the identity matrix $I \in \mathbb{R}^{n \times n}$. Given a 
matrix $P=P^{T},|v|_{P}:=\sqrt{v^{T} P v}$. Given two matrices $A$, $B$, then $A \otimes B$ denotes their Kronecker product. Given a function $(x, y) \mapsto f(x, y)$, then $\nabla_{x} f(x, y):=\left[\frac{\partial f(x, y)}{\partial x}\right]^{T}$.

\section{SETUP}

Consider two translating masses $\mathcal{Z}$ and $\mathcal{X}$ moving in an $n$-dimensional region $\mathcal{F}$ denoted by

$$
\mathcal{F}:=\left\{s \in \mathbb{R}^{2 n} \mid\left\langle F, s_{p}-s_{\circ}\right\rangle \leq 1\right\}
$$

where $F \in \mathbb{R}^{n}$ and $s_{\circ} \in \mathbb{R}^{n}$ is any point in the interior of the region. The dynamic boundary $\mathcal{J}$ of $\mathcal{F}$ is then defined by

$$
\mathcal{J}:=\left\{s \in \mathcal{F} \mid\left\langle F, s_{p}-s_{\circ}\right\rangle=1,\left\langle F, s_{v}\right\rangle \geq 0\right\}
$$

where, by $\left\langle F, s_{v}\right\rangle \geq 0, s$ belongs to $\mathcal{J}$ when the velocity subvector $s_{v}$ triggers an impact. An example is in Figure 1. We decompose each state vector $s \in \mathbb{R}^{2 n}$ into $s_{p} \in \mathbb{R}^{n}$ and $s_{v} \in \mathbb{R}^{n}$, which denote respectively position and velocity subvectors, and we call half-space the region $\mathcal{F}$ to emphasize the fact that the dynamics of $\mathcal{Z}$ and $\mathcal{X}$ resemble the behavior of two balls moving in a confined $n$-dimensional space and impacting on its boundary, corresponding to a hyperplane, which we will sometimes denote wall in the sequel.

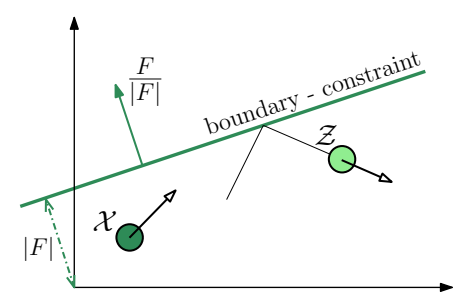

Fig. 1. Two translating masses $\mathcal{Z}$ and $\mathcal{X}$ confined, by a wall, to a half-space with $s_{\circ}=0$.

The continuous motion of the reference system is characterized by the following equations

$$
\mathcal{Z}:\left\{\begin{array}{l}
\dot{z}_{p}=z_{v} \\
\dot{z}_{v} \in \alpha(z)
\end{array}\right.
$$

where $\alpha: \mathbb{R}^{2 n} \rightrightarrows \mathbb{R}^{n}$ is a set-valued mapping that is outer semicontinuous and locally bounded, having nonempty convex values for each $z \in \mathcal{F}$. We allow for set-valued accelerations for the reference variable $z_{p}$ in order to allow for different trajectories of $z$ when starting twice from the same initial state. While the acceleration is not assumed to be unique, the selected acceleration at each time is assumed to be known by the control/estimation algorithm. When the acceleration is not assumed to be known but a bound on the acceleration is known, its effect can be typically mitigated using high feedback or observer gains.

An impact on the wall $F$ occurs when the position subvector $z_{p}$ satisfies $\left\langle F, z_{p}-s_{\circ}\right\rangle=1$ and the velocity subvector $z_{v}$ pierces the wall, that is, $\left\langle F, z_{v}\right\rangle \geq 0$. The position does not change at impacts, that is, $z_{p}^{+}=z_{p}$, while the velocity is reflected (reset) in a direction that is determined by the velocity $z_{v}$ before the impact and the wall orientation $F$. In particular, $z_{v}^{+}=M(F) z_{v}$, where

$$
\begin{aligned}
M(F) & :=R(F)^{T}\left[\begin{array}{c|c}
I_{n-1} & 0 \\
\hline 0 & -1
\end{array}\right] R(F) \\
R(F) & :=\left[\frac{F_{\perp}^{T}}{F^{T} /|F|}\right],
\end{aligned}
$$

where $F_{\perp}$ is such that $F F_{\perp}=0$, and $F_{\perp}^{T} F_{\perp}=I_{n-1}$, and $|F|=\sqrt{F^{T} F}$. Note that the columns of $F_{\perp}$ are an orthonormal basis of the orthogonal space to the vector $F$. With these definitions, $M(F)$ inverts only the component of $z_{v}$ normal to the wall $F$, by combining the matrix $\left[\begin{array}{cc}I_{n-1} & 0 \\ 0 & -1\end{array}\right]$ with the the rotation matrix $R(F)$ that maps $z_{v}$ to the base $\left\{F_{\perp}, \frac{F}{|F|}\right\}$, respectively tangential and normal directions to the wall. Thus, using the definitions

$$
\begin{aligned}
c(F) & :=F \cdot 2\left(1+F^{T} s_{\circ}\right) /|F|^{2} \\
\bar{M}(F) & :=\operatorname{diag}(M(F), M(F))=I_{2} \otimes M(F) \\
\bar{c}(F) & :=\left[\begin{array}{ll}
c(F)^{T} & 0_{1 \times n}
\end{array}\right]^{T}=\left[\begin{array}{l}
1 \\
0
\end{array}\right] \otimes c(F) \\
m(F, z) & :=\bar{M}(F) z+\bar{c}(F) .
\end{aligned}
$$

the impact dynamics can be compactly written as

$$
z^{+}=\left[\begin{array}{c}
z_{p} \\
M(F) z_{v}
\end{array}\right]=m(F, z)
$$

The next fact establishes some useful relations.

Fact 1. Given the quantities in (5),

(i) $M(F) M(F)=M(F)^{T} M(F)=I$;

(ii) $(M(F)+I) c(F)=0$;

(iii) $F^{T} M(F)=-F^{T}$;

(iv) $s=M(F) s+c(F)$ iff $\left\langle F, s-s_{\circ}\right\rangle=1$, for all $s \in \mathbb{R}^{n}$;

(v) $m(F, m(F, s))=s$, for all $s \in \mathbb{R}^{2 n}$;

(vi) $|\bar{M}(F) s|=|s|$, for all $s \in \mathbb{R}^{2 n}$.

Proof. (i) $M(F) M(F)=R(F)^{T}\left[\begin{array}{c|c}I_{n-1} & 0 \\ \hline 0 & -1\end{array}\right]^{2} R(F)=$ $R(F)^{T} R(F)=I$, and $M(F)=M(F)^{T}$. (iii) $F^{T} M(F)=$ $F^{T}\left[F_{\perp} \mid \frac{F}{|F|}\right]\left[\begin{array}{c|c}I_{n-1} & 0 \\ \hline 0 & -1\end{array}\right] R(F)=[0|-| F \mid]\left[\frac{\star}{F^{T} /|F|}\right]=$ $-F^{T}$. (ii) transposing (iii), we have $R(F) F=-F$. Moreover, denoting $c(F)=\alpha F$, we have $(M(F)+$ $I) \alpha F=\operatorname{alpha}(M(F) F+F)=0$. (iv) Rearranging, we get $s-M(F) s=\left(R(F)^{T} R(F)-M(F)\right) s=$ $\frac{1}{|F|^{2}}[\star \mid F]\left[\begin{array}{l|l}0 & 0 \\ \hline 0 & 2\end{array}\right]\left[\begin{array}{c}\star \\ \hline F^{T}\end{array}\right] s=F \frac{2 F^{T} s}{|F|^{2}}$, which coincides with $c(F)$ of (5a) if and only if $F^{T} s-F^{T} s_{\circ}=<F, s-s_{\circ}>=1$. (v) From the definitions in (5), for the velocity vector we have $s_{v}=M(F) M(F) s_{v}$ by (i) above, while for the position vector we have $M(F)\left(M(F) s_{p}+c(F)\right)+c(F)=$ $s_{p}+(M(F)+I) c(F)=s_{p}$ by (i) and (ii) above. (vi) It is sufficient to prove that $|M(F) s|=|s|$ for each $s \in \mathbb{R}^{n}$. This follows from, $|M(F) s|=\sqrt{s^{T} M(F)^{T} M(F) s}=\sqrt{s^{T} s}=$ $|s|$, by (i) above.

For reasons of control design, we restrict the motion of $\mathcal{Z}$ within a compact set $\mathcal{K}$

Assumption 1. $z$ belongs to $\mathcal{F} \cap \mathcal{K}$ where $\mathcal{K} \subset \mathbb{R}^{2 n}$ is a compact set.

Moreover, to rule out solutions that always jump and never evolve continuously, which can occur when $\mathcal{Z}$ impacts a wall with a velocity that is either zero or tangent to the 
wall $^{1}$ we augment the plant with an average dwell-time automaton Cai et al. (2008), (Goebel et al., 2009, eq. (S3), (S4)). In particular, given a positive integer $N$ and a scalar $\rho>0$, we add the dynamics

$$
\begin{aligned}
\dot{\sigma} & \in[0, \rho] & & \sigma \in[0, N] \\
\sigma^{+} & =\sigma-1 & & \sigma \in[1, N] .
\end{aligned}
$$

The arising model is hybrid Goebel et al. (2009) because the reference system $\mathcal{Z}$ evolves continuously (flows) satisfying the dynamics in (3) as long as $z \in \mathcal{K}$, while impacts (jumps) are modeled by an instantaneous reset of the state given by (6) when $z \in \mathcal{J} \cap \mathcal{K}$, and both the behaviors must be compatible with the flow and jump conditions of the dwell-time automaton in (7), that is, jumps are allowed only when $\sigma \in[1, N]$ and $z \in \mathcal{J} \cap \mathcal{K}$.

Remark 1. For a hybrid system having state in $\mathbb{R}^{2 n}$, sequences of flows and jumps which possibly characterize solutions Goebel and Teel (2006) to a hybrid system are typically denoted by functions $\xi: \operatorname{dom} \xi \rightarrow \mathbb{R}^{2 n}$, where dom $\xi$ is a subset of $\mathbb{R}_{>0} \times \mathbb{N}$ called hybrid time domain Goebel and Teel (2006), given by the union of infinitely many intervals of the form $\left[t_{j}, t_{j+1}\right] \times\{j\}$ where $0=t_{0} \leq t_{1} \leq t_{2} \leq \ldots$, or of finitely many such intervals, with the last one possibly of the form $\left[t_{j}, t_{j+1}\right] \times\{j\}$, $\left[t_{j}, t_{j+1}\right) \times\{j\}$, or $\left[t_{j}, \infty\right) \times\{j\}$. According to (Cai et al., 2008, Prop. 1.1), the dwell-time automaton (7) guarantees that any solution $\xi$ has a hybrid time domain such that for any pair $(t, j),(s, i) \in \operatorname{dom} \xi$ satisfying $t+j \geq s+i$, we have $j-i \leq \rho(t-s)+N$, namely no Zeno solution can occur. In the following neither $\rho$ nor $N$ will be used in the control design.

\section{TRACKING}

\subsection{The algorithm}

Consider a controlled system $\mathcal{X}$ which is controlled only during the continuous-time evolution and consider the goal of finding a control input $u$ for that system that guarantees asymptotic convergence of the position $x_{p}$ to the position $z_{p}$ of the reference system $\mathcal{Z}$. The dynamics of the controlled system $\mathcal{X}$ resembles that of $\mathcal{Z}$ as follows:

$$
\mathcal{X}: \dot{x}=\bar{A} x+\bar{B}(\phi(x)+u) \quad x \in \mathcal{F}
$$

where $\bar{A}=\left[\begin{array}{cc}0 & I_{n} \\ 0 & 0\end{array}\right], \bar{B}=\left[\begin{array}{c}0 \\ I_{n}\end{array}\right], \phi: \mathbb{R}^{2 n} \rightarrow \mathbb{R}^{n}$ is a continuous function representing possible nonlinear terms, and $u$ is the control input; the impact dynamics is given by

$$
\mathcal{X}: x^{+}=m(F, x) \quad x \in \mathcal{J} \text {. }
$$

When reference and controlled system are connected, the flow dynamics of the complete system is given by (8), (3) and (7a) as long as $(x, z, \sigma) \in \mathcal{C}$,

$$
\mathcal{C}:=\mathcal{F} \times \mathcal{K} \times[0, N]
$$

while the jump dynamics is given by $(9), z^{+}=z$ and $(7 \mathrm{~b})$ when $(x, z, \sigma) \in \mathcal{D}_{x}$, and by $x^{+}=x$, (6) and (7b) when $(x, z, \sigma) \in \mathcal{D}_{z}$, where

$$
\begin{aligned}
\mathcal{D}_{x} & :=\mathcal{J} \times \mathcal{K} \times[1, N], \\
\mathcal{D}_{z} & :=\mathcal{F} \times(\mathcal{J} \cap \mathcal{K}) \times[1, N], \\
\mathcal{D} & :=\mathcal{D}_{x} \cup \mathcal{D}_{z} .
\end{aligned}
$$

\footnotetext{
1 By (iii) of Fact $1,\left\langle F, s_{v}\right\rangle>0$ if and only if $\left\langle F, s_{v}^{+}\right\rangle\langle 0$, thus $s \in \mathcal{J}$
} and $s^{+} \in \mathcal{J}$ may occur only when $\left\langle F, s_{v}\right\rangle=0$.
In the absence of impacts the tracking problem can be addressed by an input $u$ that enforces convergence to zero of the $x-z$ dynamics by asymptotically stabilizing the set $\mathcal{A}_{\circ}=\{(x, z) \mid x=z\}$. However, stability may be lost in the presence of impacts, as documented in (Forni et al., 2011b, Example 1). The algorithm proposed in Forni et al. (2011b,c) recovers asymptotic tracking in the presence of impacts by using a control strategy in which $\mathcal{X}$ decides to track either the real reference or the mirrored reference (see Figure 2), based on the minimizer of a Lyapunovlike function defined as the minimum of two functions. The algorithm proposed below, instead, uses a logic variable $q$ which is updated at jumps only and replaces the minimization operation in Forni et al. (2011b,c). The implementation of the algorithm is simpler than the one in Forni et al. (2011b,c) and, following similar steps it can be generalized to polyhedral boundaries. Moreover, minor modifications to the algorithm will provide a solution for the dual problem of state estimation from the measured position output, addressed in Section 4.

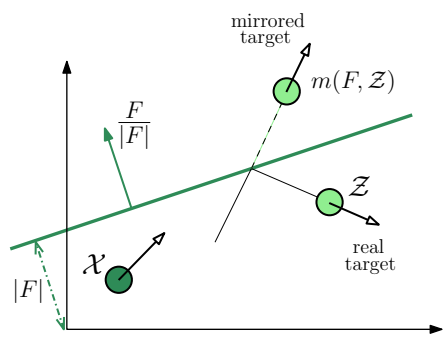

Fig. 2. Interpretation of the hybrid tracking algorithm.

The control algorithm is parameterized by a vector $K \in$ $\mathbb{R}^{n}$ satisfying the following assumption.

Assumption 2. The gain $K:=\left[\begin{array}{ll}k_{1} & k_{2}\end{array}\right]$ is such that $A_{c l}:=$ $\left[\begin{array}{cc}0 & 1 \\ k_{1} & k_{2}\end{array}\right]$ is Hurwitz.

It also uses an automaton $\mathcal{Q}$ (an index variable) toggling between 0 and 1, whose dynamics is given by

$$
\begin{array}{rlrl}
\dot{q} & =0 & & q \in\{0,1\} \\
q^{+}=1-q & q \in\{0,1\} .
\end{array}
$$

Then, by introducing the quantities

$$
M(0)=I, \quad c(0)=0, \quad \bar{K}:=K \otimes I_{n},
$$

the control law for a single wall billiard is given by

$$
u:=-\phi(x)+M(q F) \alpha+\bar{K}(x-m(q F, z))
$$

where $\alpha \in \alpha(z)$ represents the acceleration of $z_{p}$ at the current time. In particular, $x$ tracks the real target $z$ if $q=0$, when $\bar{K}(x-z)$ is enforced, and it tracks the mirrored target $m(F, z)$ if $q=1$, when $\bar{K}(x-m(F, z))$ is enforced. The tracking closed-loop system has the flow dynamics given by (8), (3), (7a), (12a), (14), which is enabled for $(x, z, \sigma, q) \in \overline{\mathcal{C}}:=\mathcal{C} \times\{0,1\} \quad$ see $(10))$, while its jump dynamics is given by the one introduced before and in (11), together with (12b), that is, by $(9), z^{+}=z,(7 \mathrm{~b}),(12 \mathrm{~b})$ for $(x, z, \sigma, q) \in \mathcal{D}_{x} \times\{0,1\}$, and by $x^{+}=x,(6)$, (7b), (12b) for $(x, z, \sigma, q) \in \mathcal{D}_{z} \times\{0,1\}$, for which the jump set is $\overline{\mathcal{D}}=\mathcal{D} \times\{0,1\}$ where $\mathcal{D}_{x}, \mathcal{D}_{z}$ and $\mathcal{D}$ are given in (11). We can now state the main result on exponential tracking.

Theorem 1. Under Assumption 2, there exist $\gamma \geq 1$ and $\lambda>0$ for which each solution $X=(x, z, \sigma, q)$ to the tracking closed-loop system satisfies 
$\left|x_{p}(t, j)-z_{p}(t, j)\right| \leq \gamma e^{-\lambda(t+j)}|x(0,0)-m(q(0,0) F, z(0,0))|$ for each $(t, j) \in \operatorname{dom} X$.

\subsection{Proof of Theorem 1}

To show that the control input (14) guarantees exponential tracking as stated in Theorem 1 , we use the compact set $\mathcal{A}$ introduced in Forni et al. (2011b,c), given by

$\mathcal{A}:=\left\{(x, z, \sigma, q) \in \mathbb{R}^{2 n} \times \mathcal{K} \times[0, N] \times\{0,1\} \mid x=m(q F, z)\right\}$

which satisfies $(x, z, \sigma, q) \in \mathcal{A} \Longrightarrow x_{p}=z_{p}$, so that stabilizing this set will enforce tracking. More specifically, to prove Theorem 1 we first recall the notion of global exponential stability and then we show that $\mathcal{A}$ is global exponentially stable (GES).

Proposition 1. Given the compact set $\mathcal{A}$ in (15), for each $X \in \overline{\mathcal{C}} \cup \overline{\mathcal{D}}$,

$$
\left|x_{p}-z_{p}\right| \leq|x-m(q F, z)| \leq R|X|_{\mathcal{A}} \leq R|x-m(q F, z)|,
$$

where $R:=\max _{z \in \mathcal{K}}\{1,|z-m(F, z)|\}$. Moreover, for each $X \in \mathcal{A} \backslash \overline{\mathcal{D}}, x=z$.

Proof. Considering $X=(x, z, \sigma, q) \in \overline{\mathcal{C}} \cup \overline{\mathcal{D}}$, which implies $(z, \sigma, q) \in \mathcal{K} \times[0, N] \times\{0,1\}$, from the definition of $\mathcal{A}$ we have

$$
\begin{aligned}
|X|_{\mathcal{A}} & =\min _{\alpha \in \mathcal{K}, \gamma \in[0, N], \beta \in\{0,1\}}||\left[\begin{array}{c}
x-m(\beta F, \alpha) \\
z-\alpha \\
\sigma-\gamma \\
q-\beta
\end{array}\right]|\leq|\left[\begin{array}{c}
x-m(q F, z) \\
0 \\
0 \\
0
\end{array}\right] \mid \\
& =|x-m(q F, z)|,
\end{aligned}
$$

which establishes the last inequality in (16). For the second inequality in (16), by using (i) $\left|v_{1}-v_{2}\right|=\mid \bar{M}(\beta F)\left(v_{1}-\right.$ $\left.v_{2}\right)|=| m\left(\beta F, v_{1}\right)-m\left(\beta F, v_{2}\right) \mid, \forall v_{1}, v_{2} \in \mathbb{R}^{2 n}, \beta \in\{0,1\}$ which follows from (5d) by (vi) of Fact 1, (ii) $\left|v_{1}-\alpha\right|^{2}+$ $\left|v_{2}-\alpha\right|^{2} \geq\left|v_{1}-v_{2}\right|^{2}, \forall v_{1}, v_{2}, \alpha \in \mathbb{R}^{2 n}$, and (iii) $R \mid q-$ $\beta|\geq| m(\beta \bar{F}, z)-m(q F, z) \mid$ which holds for all $q, \beta \in\{0,1\}$, we have

$$
\begin{aligned}
|X|_{\mathcal{A}}^{2} & =\left.\min _{\alpha \in \mathcal{K}, \gamma \in[0, N], \beta \in\{0,1\}\left|\left[\begin{array}{c}
x-m(\beta F, \alpha) \\
z-\alpha \\
\sigma-\gamma \\
q-\beta
\end{array}\right]\right|^{2}}\left|\min _{\alpha \in \mathcal{K}, \beta \in\{0,1\}}\right|\left[\begin{array}{c}
x-m(\beta F, \alpha) \\
z-\alpha \\
q-\beta
\end{array}\right]\right|^{2} \\
& =\min _{\alpha \in \mathcal{K}, \beta \in\{0,1\}}\left|\left[\begin{array}{c}
x-m(\beta F, \alpha) \\
m(\beta F, z)-m(\beta F, \alpha) \\
q-\beta
\end{array}\right]\right|^{2} \\
& =\min _{\beta \in\{0,1\}}\left|\left[\begin{array}{c}
x-m(\beta F, z) \\
q-\beta
\end{array}\right]\right|^{2} \\
& \geq \min _{\beta \in\{0,1\}} \frac{1}{R^{2}}|x-m(\beta F, z)|^{2}+|q-\beta|^{2} \\
& \geq \min _{\beta \in\{0,1\}} \frac{1}{R^{2}}\left(|x-m(\beta F, z)|^{2}+|m(\beta F, z)-m(q F, z)|^{2}\right) \\
& \geq \frac{1}{R^{2}}|x-m(q F, z)|^{2} .
\end{aligned}
$$

It follows that $|x-m(q F, z)| \leq R|X|_{\mathcal{A}}$ which establishes the second inequality in (16).

Finally, for the first inequality in (16), consider the line $\ell$ that connects $z_{p}$ to $M(F) z_{p}+c(F)$ represented by the vector $z_{p}-M(F) z_{p}-c(F)$, and note that this line is perpendicular to the wall $F$. In fact, using the definitions in (4) and (5),

$$
\begin{aligned}
F_{\perp}^{T} & \left(z_{p}-M(F) z_{p}-c(F)\right)=F_{\perp}^{T}\left((I-M(F)) z_{p}-c(F)\right) \\
& =F_{\perp}^{T}\left(R(F)^{T}\left[\frac{0}{0} \mid \frac{0}{0}\right] R(F) z_{p}-\frac{2 F}{|F|^{2}}\left(1+F^{T} s_{\circ}\right)\right) \\
& =\frac{F_{\perp}^{T}}{|F|^{2}}\left([* \mid F]\left[\begin{array}{c|c|c}
0 & 0 \\
\hline 0 & 2
\end{array}\right]\left[\frac{*}{F^{T}}\right] z_{p}-2 F\left(1+F^{T} s_{\circ}\right)\right) \\
& =\frac{F_{\perp}^{T}}{|F|^{2}}\left(2 F F^{T} z_{p}-2 F\left(1+F^{T} s_{\circ}\right)\right) \\
& =\frac{2}{|F|^{2}} F_{\perp}^{T} F\left(F^{T} z_{p}-\left(1+F^{T} s_{\circ}\right)\right)=0 .
\end{aligned}
$$

Moreover, consider the set of positions on the wall $F$ given by $\mathcal{S}_{F}:=\left\{s \in \mathbb{R}^{n} \mid F^{T}\left(s-s_{\circ}\right)=1\right\}$. We have that

$$
\left|z_{p}\right|_{\mathcal{S}_{F}}=\left|M(F) z_{p}+c(F)\right|_{\mathcal{S}_{F}} .
$$

To see this, note that $\left|z_{p}\right|_{\mathcal{S}_{F}}=\min _{\xi \in \mathcal{S}_{F}}\left|z_{p}-\xi\right|=\left|\frac{F^{T}}{|F|}\left(z_{p}-\xi\right)\right|$ for each $\xi \in \mathcal{S}_{F}$, from which $\left|z_{p}\right|_{\mathcal{S}_{F}}=\mid \frac{F^{T}}{|F|}\left(z_{p}-\xi+\right.$ $\left.s_{\circ}-s_{\circ}\right)\left|=\frac{1}{|F|}\right| F^{T}\left(z_{p}-s_{\circ}\right)-1 \mid$. Then, in a similar way, $\left|M(F) z_{p}+c(F)\right|_{\mathcal{S}_{F}}=\frac{1}{|F|} \mid F^{T}\left(M(F) z_{p}+c(F)-s_{\circ}\right)-$ $1\left|=\frac{1}{|F|}\right|-F^{T} z_{p}+2+2 F^{T} s_{0}-F^{T} s_{\circ}-1\left|=\frac{1}{|F|}\right| F^{T}\left(-z_{p}+\right.$ $\left.s_{0}\right)+\left.1|=| z_{p}\right|_{\mathcal{S}_{F}}$, where in the second identity we used (iii) of Fact 1 and the definition of $c(F)$ in (5). Consider now the set of points that belong to the line $\ell$ from $z_{p}$ to $M(F) z_{p}+c(F)$, denoted by $\mathcal{S}_{\ell}:=\left\{s \in \mathbb{R}^{n} \mid s=\lambda z_{p}+\right.$ $\left.(1-\lambda)\left(M(F) z_{p}+c(F)\right)\right\}$, and define $\eta:=\underset{s \in \mathcal{S}_{\ell}}{\operatorname{argmin}}\left|x_{p}-s\right|$, which geometrically is the point of $\mathcal{S}_{\ell}$ on the intersection between the line $\ell$ and the line perpendicular to $\ell$ passing through $x_{p}$ (which is also parallel to the wall $F$ ). We get

$$
\begin{aligned}
\left|x_{p}-z_{p}\right|^{2} & =\left|x_{p}\right|_{\mathcal{S}_{\ell}}^{2}+\left|\eta-z_{p}\right|^{2} \\
\left|x_{p}-M(F) z_{p}-c(F)\right|^{2} & =\left|x_{p}\right|_{\mathcal{S}_{\ell}}^{2}+\left|\eta-M(F) z_{p}-c(F)\right|^{2} .
\end{aligned}
$$

Then, $F^{T}\left(x_{p}-s_{\circ}\right) \leq 1$ guarantees $F^{T}\left(\eta-s_{\circ}\right) \leq 1$, from which $\left|\eta-z_{p}\right|^{2} \leq\left|z_{p}\right|_{\mathcal{S}_{F}}^{2}=\left|M(F) z_{p}+c(F)\right|_{\mathcal{S}_{F}}^{2} \leq \mid \eta-$ $M(F) z_{p}-\left.c(F)\right|^{2}$. It follows that $\left|x_{p}-z_{p}\right| \leq \mid x_{p}-M(q F) z_{p}-$ $c(q F)|\leq| x-m(q F, z) \mid$.

For the last claim of the proposition, take a point $(x, z, \sigma, q) \in \mathcal{A}$ and suppose that $x \neq z$, then $x=m(F, z)$. If $F^{T}\left(z_{p}-s_{\circ}\right)<1$ then $F^{T}\left(x_{p}-s_{\circ}\right)=F^{T}\left(M(F) z_{p}+\right.$ $\left.\left.c(F)-s_{\circ}\right)=-F^{T} z_{p}+2\left(1+F^{T} s_{\circ}\right)\right)-F^{T} s_{\circ}=-F^{T}\left(z_{p}-\right.$ $\left.s_{\circ}\right)+2>1$, where the second identity follows from (iii) of Fact 1 , thus $x$ does not belong to $\mathcal{F}$. In a similar way, if $F^{T}\left(x_{p}-s_{\circ}\right)<1$ then $F^{T}\left(z_{p}-s_{\circ}\right)>1$. The remaining case is $F^{T}\left(z_{p}-s_{\circ}\right)=F^{T}\left(x_{p}-s_{\circ}\right)=1$, and by $x=m(F, z)$ we have that $x_{v}=M(F) x_{v}$. Thus $F^{T} x_{v}=-F^{T} z_{v}$, that is, either $x$ or $z$ must belong to $\mathcal{J}$.

The following fact introduces two identities which are related to the behavior of the tracking closed-loop system at impacts. They will be used to show that the function $W$ defined next is non-increasing at jumps.

Fact 2. For any given $P=P^{T} \in \mathbb{R}^{2 \times 2}$, define $\bar{P}:=P \otimes$ $I_{n}$. For each $x, z \in \mathbb{R}^{2 n}$ and $F \in \mathbb{R}^{n}$, (i) $|x-m(F, z)|_{P}=$ $|m(F, x)-z|_{\bar{P}}$, (ii) $|m(F, x)-m(F, z)|_{\bar{P}}=|x-z|_{\bar{P}}$.

Proof. Consider the identity $\left(S_{1} \otimes S_{2}\right)\left(S_{3} \otimes S_{4}\right)=S_{1} S_{3} \otimes$ $S_{2} S_{4}$ where $S_{1}, \ldots, S_{4}$ are matrices. For each $S \in \mathbb{R}^{n \times n}$ such that $S^{T} S=I$, we have that $\left(I_{2} \otimes S^{T}\right)\left(P \otimes I_{n}\right)\left(I_{2} \otimes\right.$ $S)=\left[\left(I_{2} P\right) \otimes\left(S^{T} I_{n}\right)\right]\left(I_{2} \otimes S\right)=\left[\left(P I_{2}\right) \otimes\left(I_{n} S^{T}\right)\right]\left(I_{2} \otimes\right.$ 
$S)=\left(P \otimes I_{n}\right)\left(I_{2} \otimes S^{T}\right)\left(I_{2} \otimes S\right)=P \otimes I_{n}=\bar{P}$, thus $\bar{M}(F)^{T} \overline{P M}(F)=\bar{P}$, for each $F \in \mathbb{R}^{n}$. It follows that

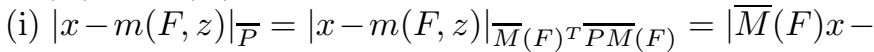
$\left.\bar{M}(F) m(F, z)\right|_{\bar{P}}=|m(F, x)-z|_{\bar{P}}$, where in the last identity we used (i) and (ii) of Fact 1; and (ii) $\mid m(F, x)-$ $\left.m(F, z)\right|_{\bar{P}}=|\bar{M}(F) x-\bar{M}(F) z|_{\bar{P}}=|x-z|_{\bar{M}(F)^{T} \overline{P M}_{(F)}}=$ $|x-z|_{\bar{P}}$.

Using the following property, we can construct a Lyapunov function $W$ which does not increase at jumps (by Fact 2) while it decreases on flows, through observability of $\left(H, A_{c l}\right)$.

Property 1. The pair of matrices $(P, H)$ are such that

$$
\begin{aligned}
& P=P^{T}>0 \\
& A_{c l}^{T} P+P A_{c l} \leq-H^{T} H \\
& \left(H, A_{c l}\right) \text { is observable }
\end{aligned}
$$

where $A_{c l}$ is defined in Assumption 2.

Remark 2. Given $A_{c l}$ Hurwitz, the results in Proposition 2 below are obtained for pairs $(P, H)$ such that $A_{c l}^{T} P+$ $P A_{c l} \leq-H^{T} H$ with $\left(H, A_{c l}\right)$ observable (according to Property 1). The generality of allowing $H^{T} H \geq 0$ instead of requiring $H^{T} H>0$ is exploited (for the case $n=2$ ) in Forni et al. (2011a) to analyze some specific multiplewall billiards (parallel walls) for which the nonincreasing feature of the function $W$ at jumps cannot be guaranteed by a matrix $P$ which satisfies the stronger condition $A_{c l}^{T} P+$ $P A_{c l}<0$.

Note that if Assumption 2 holds, there always exists a pair $(P, H)$ that satisfy Property 1 . Thus, using

$$
\bar{P}:=P \otimes I_{n}, \quad \bar{H}:=H \otimes I_{n},
$$

we can establish the following result:

Proposition 2. If Property 1 holds, consider the function $W: \overline{\mathcal{C}} \cup \overline{\mathcal{D}} \rightarrow \mathbb{R}_{\geq 0}$ given by

$$
W(x, z, \sigma, q)=|x-m(q F, z)| \frac{2}{P} .
$$

Then, using $X=(x, z, \sigma, q)$ and $R:=\max _{z \in \mathcal{K}}\{1,|z-m(F, z)|\}$

(i) $\lambda_{\min }(P)|X|_{\mathcal{A}}^{2} \leq W(X) \leq \lambda_{\max }(P) R^{2}|X|_{\mathcal{A}}^{2}, \forall X \in \overline{\mathcal{C}} \cup \overline{\mathcal{D}}$;

(ii) $\dot{W}(X) \leq-|x-m(q F, z)|_{\bar{H}^{T}}^{2}, \forall X \in \overline{\mathcal{C}}$;

(iii) $W\left(X^{+}\right) \leq W(X), \forall X \in \overline{\mathcal{D}}$.

Proof. From the definition of $W, \lambda_{\min }(P)|x-m(q F, z)|^{2} \leq$ $W(X) \leq \lambda_{\max }(P)|x-m(q F, z)|^{2}$. Then, (i) follows from Proposition 1 .

For (ii), using $\bar{A} \bar{c}(q F)=0$ and $\bar{M}(q F) \bar{A}=\left(I_{2} \otimes\right.$ $M(q F))\left(\left[\begin{array}{ll}0 & 1 \\ 0 & 0\end{array}\right] \otimes I_{n}\right)=\left(I_{2}\left[\begin{array}{ll}0 & 1 \\ 0 & 0\end{array}\right]\right) \otimes\left(M(q F) I_{n}\right)=\left(\left[\begin{array}{ll}0 & 1 \\ 0 & 0\end{array}\right] I_{2}\right) \otimes$ $\left(I_{n} M(q F)\right)=\left(\left[\begin{array}{ll}0 & 1 \\ 0 & 0\end{array}\right] \otimes I_{n}\right)\left(I_{2} \otimes M(q F)\right)=\overline{A M}(q F)$ in the second identity, we get

$$
\begin{aligned}
\dot{W} & (X)=2(x-m(q F, z))^{T} \bar{P}(\bar{A} x-\bar{M}(q F) \bar{A} z \\
& +\overline{B K}(x-m(q F, z))) \\
& =2(x-m(q F, z))^{T} \bar{P}(\bar{A}+\overline{B K})(x-m(q F, z)) \\
& =2(x-m(q F, z))^{T}\left(P A_{c l} \otimes I_{n}\right)(x-m(q F, z)) \\
& =-|x-m(q F, z)|_{H^{T}} \bar{H}^{2} .
\end{aligned}
$$

(iii) Consider a jump of $\mathcal{Z}$. Using (v) of Fact 1 for $q=0$, and $m(0, m(F, z))=m(F, z)=m(q F, z)$ for $q=1$ (which follows from $M(0)=I$ and $c(0)=0)$, we get

$$
\begin{aligned}
\left|x^{+}-m\left(q^{+} F, z^{+}\right)\right|_{\bar{P}} & =|x-m((1-q) F, m(F, z))|_{\bar{P}} \\
& =\mid x-m(q F, z))\left.\right|_{\bar{P}} .
\end{aligned}
$$

Consider a jump of $\mathcal{X}$. From (i) of Fact 2, and by using the argument above, we get

$$
\begin{aligned}
\left|x^{+}-m\left(q^{+} F, z^{+}\right)\right|_{\bar{P}} & =|m(F, x)-m((1-q) F, z)|_{\bar{P}} \\
& =\mid x-m(q F, z))\left.\right|_{\bar{P}} .
\end{aligned}
$$

The results in Proposition 2 will be used in the proof of the next theorem to establish global exponential stability of the set $\mathcal{A}$. Then, Theorem 1 follows from Proposition 1 and global exponential stability of $\mathcal{A}$.

Definition 1. Consider a hybrid system $\mathcal{H}$ with state $X \in$ $\mathbb{R}^{2 n}$ and a compact set $\mathcal{A} \subseteq \mathbb{R}^{2 n}$. We say that $\mathcal{A}$ is globally exponentially stable (GES) if there exist $\gamma \geq 1$ and $\lambda>0$ such that each solution $X$ to $\mathcal{H}$ satisfies $|X(t, j)|_{\mathcal{A}} \leq \gamma e^{-\lambda(t+j)}|X(0,0)|_{\mathcal{A}}$ for all $(t, j) \in \operatorname{dom} X$.

Theorem 2. Under Assumption 2, for each $\rho>0$ and $N>0$, the compact set $\mathcal{A}$ is globally exponentially stable for the tracking closed-loop system.

The proof of Theorem 2 is based on the following Lemma which is a reformulation of (Teel et al., 2011, Theorem 2) (see also the proof of (Teel et al., 2011, Theorem 2)).

Lemma 1. Consider an observable pair $(H, A)$, with $A \in$ $\mathbb{R}^{n \times n}$, a map $G: \mathbb{R}^{2 n} \times \mathbb{R}^{m} \rightrightarrows \mathbb{R}^{2 n}$, and two sets $\mathcal{E}_{c} \subseteq \mathbb{R}^{2 n}$, $\mathcal{E}_{d} \subseteq \mathbb{R}^{2 n} \times \mathbb{R}^{m}$. Suppose that there exists a function $V: \mathbb{R}^{2 n} \rightarrow \mathbb{R}_{\geq 0}$ defined as $V(e):=|e|_{P}^{2}$ for all $e \in \mathbb{R}^{2 n}$, with $P \in \mathbb{R}^{n \times n}$ symmetric and positive definite, satisfying:

$\left(a_{1}\right)\langle\nabla V(e), A e\rangle \leq-|e|_{H^{T} H}^{2} \quad \forall e \in \mathcal{E}_{c}$;

$\left(a_{2}\right) V(g) \leq V(e) \quad \forall(e, \xi) \in \mathcal{E}_{d}, \forall g \in G(e, \xi)$.

Then, for each pair $(\rho, N) \in \mathbb{R}_{>0} \times \mathbb{Z}_{>0}$, there exists a function $Y: \mathbb{R}^{2 n} \times[0, N] \rightarrow \mathbb{R}_{\geq 0}$ and scalars $\lambda_{1}, \lambda_{2}, \lambda_{3}>0$, $\lambda_{4} \in[0,1)$, such that

(i) $\lambda_{1}|e|^{2} \leq Y(e, \sigma) \leq \lambda_{2}|e|^{2}, \forall \sigma \in[0, N], \forall e \in \mathbb{R}^{2 n}$

(ii) $\left\langle\nabla_{\sigma} Y(e, \sigma), f\right\rangle+\left\langle\nabla_{e} Y(e, \sigma), A e\right\rangle \leq-\lambda_{3} Y(e, \sigma), \forall \sigma \in$ $[0, N], \forall e \in \mathcal{E}_{c}, \forall f \in[0, \rho]$

(iii) $Y(g, \sigma-1) \leq \lambda_{4} Y(e, \sigma), \forall \sigma \in[1, N], \forall(e, \xi) \in$ $\mathcal{E}_{d}, \forall g \in G(e, \xi)$

Proof of Theorem 2. Under Assumption 2 we can find a pair of matrices $(P, H)$ which satisfy Property 1 . Then, if we only need to establish global asymptotic stability, this property follows from Proposition 2, observability of $\left(H, A_{c l}\right)$, the average dwell-time constraint imposed by $(7)$, and the invariance principle Sanfelice et al. (2007a). Since we need to establish global exponential stability, we invoke Lemma 1. In particular, consider the coordinate transformation $(e, \sigma, \xi):=(x-m(q F, z), \sigma,(z, q))$. Then, for each solution $X=(x, z, \sigma, q)$ to the tracking closed loop system, using the new coordinates and Proposition 2, define $V(e)=W(X)$ and note that $\dot{e}=A_{c l} \otimes I_{n}, V(e)=W(X)=$ $|e| \frac{2}{P}, \dot{V}(e)=\dot{W}(X)=\left\langle\nabla V(e), A_{c l} \otimes I_{n}\right\rangle \leq-|e|_{\bar{H}^{T}}^{2}{ }_{H}$ on flows and $V\left(e^{+}\right)=W\left(X^{+}\right) \leq W(X) \leq V(e)$ across jumps. Thus, choosing $A$ in Lemma 1 as $A_{c l} \otimes I_{n}$, and by a suitable definitions of $G, \mathcal{E}_{c}$ and $\mathcal{E}_{d}$, each condition of Lemma 1 is satisfied. Therefore, from $(i)$-(iii) of Lemma 1 and by (i) of Proposition 1 , defining $\bar{Y}(X):=Y(e, \sigma)=Y(x-$ $m(q F, z), \sigma)$ and $R:=\max _{z \in \mathcal{K}}\{1,|z-m(F, z)|\}$, we get 
- $\lambda_{1}|X|_{A}^{2} \leq \lambda_{1}|x-m(q F, z)|^{2} \leq \bar{Y}(X) \leq \lambda_{2} \mid x-$ $\left.m(q F, z)\right|^{2} \leq \sqrt{2} R \lambda_{2}|X|_{\mathcal{A}}^{2}, \forall X \in \overline{\mathcal{C}} \cup \overline{\mathcal{D}}$

- $\dot{\bar{Y}}(X) \leq-\lambda_{3} \bar{Y}(X), \forall X \in \overline{\mathcal{C}}$

- $\bar{Y}\left(X^{+}\right) \leq \lambda_{4} \bar{Y}(X), \forall X \in \overline{\mathcal{D}}$,

which, according to (Teel et al., 2011, Theorem 1), establish global exponential stability of $\mathcal{A}$.

Remark 3. Suppose that $X=(x, z, \sigma, q)$ belongs to $(\mathcal{A}+$ $\varepsilon \mathbb{B}) \cap \overline{\mathcal{C}} \cup \overline{\mathcal{D}}$ with $\varepsilon>0$. Then, when $\varepsilon$ is (sufficiently) small, the control algorithm proposed above is equivalent to the control algorithm proposed in (Forni et al., 2011b, Section III), which is defined by a Lyapunov-based selection of the real/mirrored targets and enforces local asymptotic stability of $\mathcal{A}$.

Remark 4. The hybrid dynamics of the two translating masses, the control algorithm presented in Sections 2 and 3 , and the analysis performed above can be particularized to well known situations for specific values of the space dimension $n$. In particular, for the case $n=1$, the system corresponds to a bouncing ball hitting a wall in a one dimensional space, under the action of a (possibly nonlinear) acceleration $\alpha(z)$ which would be $\alpha(z)=-g$ for the standard bouncing ball. For the case $n=2$ the system resembles a ball moving on a surface and hitting against a straight edge, similar to the case addressed in Forni et al. (2011b) therein associated with billiards. Finally, if $n=3$, the system corresponds to a ball moving in the threedimensional space and hitting against a wall having an arbitrary orientation.

\section{STATE ESTIMATION}

\subsection{The algorithm}

We consider the problem of designing an observer $\mathcal{X}$ to estimate the state of $\mathcal{Z}$ from the output $y=C z$, $C:=\left[I_{n} \mid 0\right] \in \mathbb{R}^{n \times 2 n}$, i.e. from the measurement of the position $z_{p}$ only.

We replace the continuous dynamics (3) of the reference system $\mathcal{Z}$ by

$$
\mathcal{Z}: \dot{z} \in \bar{A} z+\bar{B} \alpha(C z) \quad z \in \mathcal{K}
$$

where $\alpha: \mathbb{R}^{n} \rightrightarrows \mathbb{R}^{n}$ is now an outer semicontinuous and locally bounded set-valued map having nonempty convex values for each $z_{p}=C z \in \Pi_{p}(\mathcal{F}):=\left\{z_{p} \mid z \in \mathcal{F}\right\}$.

The observer continuous dynamics is given by

$$
\mathcal{X}: \dot{x}=\bar{A} x+u \quad x \in \mathcal{F}
$$

where, different from Section 3, the action of $u=$ $\left[u_{p}^{T} \mid u_{v}^{T}\right]^{T} \in \mathbb{R}^{2 n}$ now affects both $\dot{x}_{p}$ and $\dot{x}_{v}$. The jump dynamics of the observer resembles the impact dynamics of the tracking case, and is given by

$$
\mathcal{X}: x^{+}=m(F, x) \quad\left(x_{p}, x_{v}+u_{p}\right) \in \mathcal{J},
$$

which differs from (9) only for the definition of the jump set, which presents an explicit dependence on the input subvector $u_{p}$. In fact, the dynamics $\dot{x}_{p}=x_{v}$ of the tracking approach is now replaced by $\dot{x}_{p}=x_{v}+u_{p}$, from which the impact condition $\left\langle F, \dot{x}_{p}\right\rangle=\left\langle F, x_{v}\right\rangle \geq 0$ is replaced by $\left\langle F, \dot{x}_{p}\right\rangle=\left\langle F, x_{v}+u_{p}\right\rangle \geq 0$.

Remark 5. Although the jump dynamics of the observer is not necessarily connected to the impacts physics of the billiard (no "physical" walls are impacted by the observer), with the new definition of the jump set, we preserve the analogy with the tracking case, enforcing a reset behavior of the observer that resembles the impact behavior of a translating mass whose velocity is given by $\dot{x}_{p}=s_{1}+s_{2}$, with $s_{1}=x_{v}$ and $s_{2}=u_{p}$. Note that when $u_{p}=0$, the jump dynamics of $\mathcal{X}$ (both the jump set and the jump map) coincides with the jump dynamics of $\mathcal{Z}$.

Following the approach of Section 3, the observer algorithm is parameterized by a vector $L \in \mathbb{R}^{n}$ satisfying the following assumption.

Assumption 3. The gain $L=\left[\begin{array}{ll}\ell_{1} & \ell_{2}\end{array}\right]^{T}$ is such that $A_{c l}:=$ $\left[\begin{array}{ll}\ell_{1} & 1 \\ \ell_{2} & 0\end{array}\right]$ is Hurwitz.

Thus, using (4) and (5) and $\bar{L}:=L \otimes I_{n}$, the input $u$ for a single wall billiard is given by

$$
\begin{aligned}
u & :=\bar{M}(q F) \bar{B} \alpha+\bar{L}(C x-M(q F) C z-c(q F)) \\
& =\bar{M}(q F) \bar{B} \alpha+\bar{L} C(x-m(q F, z)),
\end{aligned}
$$

where $\alpha \in \alpha(C z)$ represents the acceleration of $z_{p}$ at the current time, and the observer closed-loop system has the flow dynamics given by (28), (27), (7a), (12a), and (30), which is enabled for $(x, z, \sigma, q) \in \overline{\mathcal{C}}$, where

$$
\overline{\mathcal{C}}:=\mathcal{F} \times \mathcal{K} \times[0, N] \times\{0,1\},
$$

while the jump dynamics is given by $(29), z^{+}=z,(7 \mathrm{~b})$, (12b) when $\left(\left(x_{p}, x_{v}+u_{p}\right), z, \sigma, q\right) \in \overline{\mathcal{D}}_{x}$, and by $x^{+}=x$, (6), (7b), (12b) when $(x, z, \sigma, q) \in \overline{\mathcal{D}}_{z}$, where

$$
\begin{aligned}
\overline{\mathcal{D}}_{x} & :=\mathcal{J} \times \mathcal{K} \times[1, N] \times\{0,1\}, \\
\overline{\mathcal{D}}_{z} & :=\mathcal{F} \times(\mathcal{J} \cap \mathcal{K}) \times[1, N] \times\{0,1\}, \\
\overline{\mathcal{D}} & =\overline{\mathcal{D}}_{x} \cup \overline{\mathcal{D}}_{z} .
\end{aligned}
$$

\subsection{Closed-loop results}

Following the approach of Section 3.2, we state below the main result of the current section, on stability of the set $\mathcal{A}$ defined in (15).

Theorem 3. Under Assumption 3, for each $\rho>0$ and $N>0$, the compact set $\mathcal{A}$ is globally exponentially stable for the observer closed-loop system.

Proof. Under Assumption 3, consider a pair of matrices $(P, H)$ which satisfy $(21)$ with $A_{c l}=\left[\begin{array}{ll}\ell_{1} & 1 \\ \ell_{2} & 0\end{array}\right]$ and define $W: \overline{\mathcal{C}} \cup \overline{\mathcal{D}} \rightarrow \mathbb{R}_{\geq 0}$ given by $W(x, z, \sigma, q)=|x-m(q F, z)| \frac{2}{P}$. Then, the function $W$ above and the jump dynamics of the observer closed-loop system satisfy statements (i) and (iii) of Proposition 2, which can be established by following the same arguments proposed at points (i) and (iii) of the proof of Proposition 2. Moreover, following (ii) of the proof of Proposition 2, using the relations $\bar{A} \bar{c}(q F)=0$ and $\bar{M}(q F) \bar{A}=\overline{A M}(q F)$ in the second identity below, we get

$$
\begin{aligned}
\dot{W}(X)= & 2(x-m(q F, z))^{T} \bar{P}(\bar{A} x+\bar{L}(C x-C m(q F, z)) \\
& -\bar{M}(q F) \bar{A} z) \\
= & 2(x-m(q F, z))^{T} \bar{P}(\bar{A}+\bar{L} C)(x-m(q F, z)) \\
\leq & 2(x-m(q F, z))^{T}\left(P A_{c l} \otimes I_{n}\right)(x-m(q F, z)) \\
\leq & -|x-m(q F, z)|_{H^{T}}^{2} \bar{H}^{2} .
\end{aligned}
$$

Then, global exponential stability follows from Lemma 1 and (Teel et al., 2011, Theorem 1) using the coordinate transformation $(e, \sigma, \xi):=(x-m(q F, z), \sigma,(z, q))$, as in the proof of Theorem 2 . 
The combination of the jump set in (29) and of $u$ in (30) guarantees that if $\left(x_{p}, x_{v}+u_{p}\right) \in \mathcal{J}$ with $\left\langle F, x_{v}+\right.$ $\left.u_{p}\right\rangle>0$, then $\left(x_{p}, x_{v}+u_{p}\right)^{+} \notin \mathcal{J}$, as established in the next proposition, thus it guarantees that no Zeno solutions are induced by the observer algorithm. In fact, the dwelltime automaton $\sigma$ has been introduced in Section 2 to rule out trajectories that impact a wall with null normal component, i.e. $\left\langle F, \dot{x}_{p}\right\rangle=\left\langle F, x_{v}+u_{p}\right\rangle=0$, which is usually associated with a translating mass sliding along the wall, and for which the arising Zeno phenomena can be essentially considered as a mathematical side-effect of the particular model adopted. Proposition 3 guarantees that also for the observer closed-loop system the dwelltime automaton only operates on those trajectories, since the jump dynamics (29) does not introduce new Zeno phenomena.

Proposition 3. For the observer closed-loop system, if $\left\langle F, x_{p}-s_{\circ}\right\rangle=1$ and $\left\langle F, x_{v}+u_{p}\right\rangle>0$ then $\left\langle F,\left(x_{v}+\right.\right.$ $\left.\left.u_{p}\right)^{+}\right\rangle<0$.

Proof. Suppose that $F^{T}\left(x_{p}-s_{\circ}\right)=1$ and $F^{T}\left(x_{v}+\right.$ $\left.u_{p}\right)=F^{T}\left(x_{v}+\ell_{1}\left[x_{p}-M(q F) z_{p}-c(q F)\right]\right)>0$. In this case, $x_{p}^{+}=M(F) x_{p}+c(F)=x_{p}$ (by (iv) of Fact 1) and $z_{p}^{+}=z_{p}$ (no jump). For the case $q=0, q^{+}=1$, using $M(q F)=M(0)=I$ and $c(q F)=c(0)=0$ in the next to last identity, and (iii) of Fact 1 in the last identity, we have

$$
\begin{aligned}
F^{T} & \left.x_{v}+u_{p}\right)^{+} \\
& =F^{T}\left(x_{v}^{+}+\ell_{1}\left[x_{p}^{+}-M\left(q^{+} F\right) z_{p}^{+}-c\left(q^{+} F\right)\right]\right) \\
& =F^{T}\left(M(F) x_{v}+\ell_{1}\left[M(F) x_{p}+c(F)-M(F) z_{p}-c(F)\right]\right) \\
& =F^{T} M(F)\left(x_{v}+\ell_{1}\left[x_{p}-z_{p}\right]\right) \\
& =F^{T} M(F)\left(x_{v}+\ell_{1}\left[x_{p}-M(q F) z_{p}-c(q F)\right]\right) \\
& =-F^{T}\left(x_{v}+u_{p}\right)
\end{aligned}
$$

For the case $q=1, q^{+}=0$, using in the third identity (i) of Fact 1 and $F^{T} c(F)=-F^{T} M(F) c(F)$ (by (iii) of Fact 1), we have

$$
\begin{aligned}
F^{T} & \left(x_{v}+u_{p}\right)^{+}=F^{T}\left(x_{v}^{+}+\ell_{1}\left[x_{p}^{+}-M\left(q^{+} F\right) z_{p}^{+}-c\left(q^{+} F\right)\right]\right) \\
& =F^{T}\left(M(F) x_{v}+\ell_{1}\left[M(F) x_{p}+c(F)-z_{p}\right]\right) \\
& =F^{T} M(F)\left(x_{v}+\ell_{1}\left[x_{p}-c(F)-M(F) z_{p}\right]\right) \\
& =F^{T} M(F)\left(x_{v}+\ell_{1}\left[x_{p}-M(q F) z_{p}-c(q F)\right]\right) \\
& =-F^{T}\left(x_{v}+u_{p}\right)
\end{aligned}
$$

Summarizing, Theorem 3 establishes global exponential stability of $\mathcal{A}$ which, by Proposition 1, corresponds to the set where $x=z$ (namely zero observation error), except for the hybrid times when jumps occur. ${ }^{2}$ Moreover, Proposition 3 guarantees that when the observer mass $\mathcal{X}$ impacts a wall with speed $\dot{x}_{p}$ piercing its boundary, after the arising jump, the mass is reflected back toward the interior of the billiard $\mathcal{F}$.

\footnotetext{
2 Indeed, at those times, $\mathcal{A}$ allows for an instantaneous mismatch of the speeds $\left(x_{v}=M(F) z_{v} \neq z_{v}\right)$ arising from a pair of consecutive jumps occurring at the same ordinary time $t$. For example, if $\mathcal{Z}$ jumps first, then $\left(x_{v}(t, j), z_{v}(t, j)\right)=\left(\bar{z}_{v}, \bar{z}_{v}\right) \rightarrow\left(x_{v}(t, j+1), z_{v}(t, j+1)\right)=$ $\left(\bar{z}_{v}, M(F) \bar{z}_{v}\right) \rightarrow\left(x_{v}(t, j+2), z_{v}(t, j+2)\right)=\left(M(F) \bar{z}_{v}, M(F) \bar{z}_{v}\right)$
}

\section{OUTPUT FEEDBACK}

In this section we combine the tracking and state estimation algorithms of the previous sections to construct an output feedback controller. We consider the following setup: $\mathcal{Z}$ is the exogenous system and we have full access to its state, $\mathcal{X}$ is the controlled system (the plant) and we measure its position $y=C x$, and $\widehat{\mathcal{X}}$ is the dynamic controller whose output drives $\mathcal{X}$ to achieve asymptotic tracking of $\mathcal{Z}$. The continuous dynamics of the closed-loop system is given by

$$
\begin{array}{ll}
\mathcal{Z}:\left\{\dot{z}=\bar{A} z+\bar{B} d_{1}\right. & \\
\mathcal{X}:\left\{\dot{x}=\bar{A} x+\bar{B}\left(d_{2}+u_{c}\right),\right. & y=C x \\
\widehat{\mathcal{X}}:\left\{\dot{\hat{x}}=\bar{A} \hat{x}+u_{o}, \quad \dot{q}=0,\right. & \dot{\hat{q}}=0
\end{array}
$$

and by (7a), where, for simplicity of notation, $d_{1}$ and $d_{2}$ are signals measured by the dynamic controller, possibly replacing functions like $\alpha$ in (3) and $\phi$ in (8), used in the previous sections. Here, $u_{c}$ and $u_{o}$ are defined by

$$
\begin{aligned}
& u_{c}=M(q F) d_{1}+\bar{K}(m(\hat{q} F, \hat{x})-m(q F, z)), \\
& u_{o}=\bar{M}(\hat{q} F) \bar{B}\left(d_{2}+u_{c}\right)+\bar{L}[C \hat{x}-C m(\hat{q} F, x)],
\end{aligned}
$$

where $\bar{K} \in \mathbb{R}^{n \times 2 n}$ and $\bar{L} \in \mathbb{R}^{2 n \times n}$ are respectively the controller and the observer gains. The overall state is defined as $X=(z, x, \hat{x}, q, \hat{q}, \sigma)$ and the flow set is given by

$$
\overline{\mathcal{C}}:=\mathcal{K} \times \mathcal{F} \times \mathcal{F} \times\{0,1\} \times\{0,1\} \times[0, N] .
$$

The discrete dynamics is given by

$$
\left\{\begin{array} { l } 
{ z ^ { + } \in m ( F , z ) } \\
{ x ^ { + } = x } \\
{ \hat { x } ^ { + } = \hat { x } } \\
{ q ^ { + } = q - 1 } \\
{ \hat { q } ^ { + } = \hat { q } } \\
{ \sigma ^ { + } = \sigma - 1 , }
\end{array} \quad \left\{\begin{array} { l } 
{ z ^ { + } = z } \\
{ x ^ { + } = m ( F , x ) } \\
{ \hat { x } ^ { + } = \hat { x } } \\
{ q ^ { + } = q - 1 } \\
{ \hat { q } ^ { + } = \hat { q } - 1 } \\
{ \sigma ^ { + } = \sigma - 1 , }
\end{array} \quad \left\{\begin{array}{l}
z^{+}=z \\
x^{+}=x \\
\hat{x}^{+}=m(F, \hat{x}) \\
q^{+}=q \\
\hat{q}^{+}=\hat{q}-1 \\
\sigma^{+}=\sigma-1
\end{array}\right.\right.\right.
$$

respectively for $X \in \overline{\mathcal{D}}_{z}, X \in \overline{\mathcal{D}}_{x}$, and $X \in \overline{\mathcal{D}}_{\hat{x}}$, with those sets defined as

$$
\begin{aligned}
\overline{\mathcal{D}}_{z} & :=\{X \in \overline{\mathcal{C}} \mid z \in \mathcal{J}, \sigma \in[1, N]\}, \\
\overline{\mathcal{D}}_{x} & :=\{X \in \overline{\mathcal{C}} \mid x \in \mathcal{J}, \sigma \in[1, N]\}, \\
\overline{\mathcal{D}}_{\hat{x}} & :=\left\{X \in \overline{\mathcal{C}} \mid\left(\hat{x}_{p}, \hat{x}_{v}+u_{o, p}\right) \in \mathcal{J}, \sigma \in[1, N]\right\}, \\
\overline{\mathcal{D}} & :=\overline{\mathcal{D}}_{z} \cup \overline{\mathcal{D}}_{x} \cup \overline{\mathcal{D}}_{\hat{x}},
\end{aligned}
$$

where the vector $u_{o, p}$ used in the definition of the jump set above is given by $u_{o}=\left[u_{o, p}^{T} \mid u_{o, v}^{T}\right]^{T}$ (this arises from the observer construction in Section 4). Note that $q$ is updated when either $\mathcal{Z}$ or $\mathcal{X}$ jumps, while $\hat{q}$ is updated when either $\mathcal{X}$ or $\widehat{\mathcal{X}}$ jumps (since $\mathcal{X}$ plays here the role of the exogenous system whose state is estimated by the observer $\widehat{\mathcal{X}})$. The next stability result is established by focusing on the set:

$$
\mathcal{A}:=\left\{(z, x, \hat{x}, q, \hat{q}, \sigma) \in \Pi \mid x=m_{q}(z), \hat{x}=m_{\hat{q}}(x)\right\},
$$

where $\Pi:=\mathcal{K} \times \mathbb{R}^{2 n} \times \mathbb{R}^{2 n} \times\{0,1\} \times\{0,1\} \times[0, N]$, which parallels the set $\mathcal{A}$ defined in (15). It is also based on the next assumption which extends to the output feedback case the assumptions of the previous sections.

Assumption 4. The exosystem $\mathcal{Z}$ is restricted to a compact set $\mathcal{K}$ which satisfies Assumption $1 ; K$ satisfies Assumption 2 and $\bar{K}:=K \otimes I_{n} ; L$ satisfies Assumption 3 and $\bar{L}:=L \otimes I_{n}$. 
For the set $\mathcal{A}$ in (39) we can follow similar steps to those of the proof of Proposition 1 and establish the bounds stated next.

Proposition 4. Given $\mathcal{A}$ in (39), there exists a scalar $R>0$ such that for each $X=(z, x, \hat{x}, q, \hat{q}, \sigma) \in \overline{\mathcal{C}} \cup \overline{\mathcal{D}}$,

$$
\begin{aligned}
|X|_{\mathcal{A}} & \geq \frac{1}{\sqrt{6} R}\left(\left|x-m_{q}(z)\right|+\left|\hat{x}-m_{\hat{q}}(z)\right|\right) \\
|X|_{\mathcal{A}} & \leq \sqrt{2}\left(\left|x-m_{q}(z)\right|+\left|\hat{x}-m_{\hat{q}}(z)\right|\right) .
\end{aligned}
$$

Thus, establishing global exponential stability of the set $\mathcal{A}$ in (39) will guarantee output feedback tracking.

Theorem 4. Under Assumptions 4, consider a pair $\left(P_{c}, H_{c}\right)$ which satisfies (21) for $A_{c l}=\left[\begin{array}{cc}0 & 1 \\ k_{1} & k_{2}\end{array}\right]$, a pair $\left(P_{o}, H_{o}\right)$ which satisfies (21) for $A_{c l}=\left[\begin{array}{ll}\ell_{1} & 1 \\ \ell_{2} & 0\end{array}\right]$, and define $\bar{P}_{c}=P_{c} \otimes$ $I_{n}, \bar{P}_{o}=P_{o} \otimes I_{n}$. Then, for each $\rho>0$ and $N>0, \mathcal{A}$ in (39) is GES for the closed-loop system.

Proof sketch. Define $e_{1}:=\hat{x}-m(\hat{q} F, x)$ and $e_{2}:=x-$ $m(q F, z)$. Using similar identities to those exploited in Propositions 2 and 3 , it is possible to show that the flow dynamics of $e:=\left(e_{1}, e_{2}\right)$ corresponds to

$$
\dot{e}=\left[\begin{array}{c|c}
\bar{A}+\bar{L} C & 0 \\
\hline-\overline{B K M}(\hat{q} F) & \bar{A}+\overline{B K}
\end{array}\right] e .
$$

Define now $W_{o}\left(e_{1}\right):=\left|e_{1}\right| \frac{2}{P_{o}}$ and $W_{c}\left(e_{2}\right):=\left|e_{2}\right| \frac{2}{P_{c}}$. From Assumption 3, we have $\dot{W}_{o}\left(e_{1}\right) \leq\left|e_{1}\right| \frac{2}{H_{o}}{ }^{T} \bar{H}_{o}$ during flows, where the matrix $\bar{H}_{o}=H_{o} \otimes I_{2}$ guarantees $\left(\bar{H}_{o}, \bar{A}+\right.$ $\bar{L} C)$ observable. Moreover, following similar derivations to the proof of item (iii) of Proposition 2 (in particular, see equations $(25)$ and $(26))$ we have $W_{o}\left(e_{1}^{+}\right) \leq W_{o}\left(e_{1}\right)$ at jumps. Thus, using the coordinates $\left(e_{1}, \sigma,(x, z, q, \hat{q})\right)$ and Lemma 1 ( $A$ in Lemma 1 is given in this case by $\bar{A}+\bar{L} C)$, we get the function $Y_{o}\left(e_{1}, \sigma\right)$ which satisfies $(i)$ (iii) of Lemma 1. In a similar way, when $e_{1}=0$, we have $\dot{W}_{c}\left(e_{2}\right) \leq\left|e_{2}\right|_{\bar{H}_{c}}{ }^{T} \bar{H}_{c}$ during flows, where $\bar{H}_{c}=H_{c} \otimes I_{2}$ and the pair $\left(\bar{H}_{c}, \bar{A}_{c}+\overline{B K}\right)$ is observable, and following similar derivations to the proof of item (iii) of Proposition 2 we have $W_{c}\left(e_{2}^{+}\right) \leq W_{c}\left(e_{2}\right)$ at jumps. So, for the coordinates $\left(e_{2}, \sigma,\left(z, q, \hat{q}, e_{1}\right)\right)$, supposing $e_{1}=0$, by Lemma 1 , we get the function $Y_{c}\left(e_{2}, \sigma\right)$ which satisfies $(i)$ - $(i i i)$ of Lemma 1.

Define now $V(X):=\bar{\rho} Y_{o}\left(e_{1}, \sigma\right)+Y_{c}\left(e_{2}, \sigma\right)$, where $\bar{\rho}>0$ and $X=(z, x, \hat{x}, q, \hat{q}, \sigma)$. Note that $V$ is positive definite w.r.t $\mathcal{A}$. Then, using standard Lyapunov derivations for cascaded systems, there exists a large enough $\bar{\rho}$ and a constant $\gamma_{f}>0$ such that

$$
\dot{V}(X) \leq-\gamma_{f} V(X), \quad \forall X \in \overline{\mathcal{C}}
$$

Moreover, from the properties of $Y_{c}$ and $Y_{o}$ across jumps, there exists $\gamma_{g} \in[0,1)$ such that

$$
V\left(X^{+}\right) \leq \gamma_{g} V(X), \quad \forall X \in \overline{\mathcal{D}}
$$

Then, also using the bounds in Proposition 4, by (Teel et al., 2011, Theorem 1) the set $\mathcal{A}$ is GES.

\section{REFERENCES}

Brogliato, B., 2004. Absolute stability and the LagrangeDirichlet theorem with monotone multivalued mappings. Systems \& control letters 51 (5), 343-353.

Cai, C., Teel, A., Goebel, R., 2008. Smooth Lyapunov functions for hybrid systems Part II:(pre) asymptoti- cally stable compact sets. IEEE Transactions on Automatic Control 53 (3), 734-748.

Forni, F., Teel, A., Zaccarian, L., 2011a. Follow the bouncing ball: global results on tracking and state estimation with impacts. IEEE Trans. Aut. Cont., submitted.

Forni, F., Teel, A., Zaccarian, L., Dec. 2011b. Tracking control in billiards using mirrors without smoke, part I: Lyapunov-based local tracking in polyhedral regions. In: Joint CDC-ECC. Orlando (FL), USA.

Forni, F., Teel, A., Zaccarian, L., Dec. 2011c. Tracking control in billiards using mirrors without smoke, part II: additional Lyapunov-based local and global results. In: Joint CDC-ECC. Orlando (FL), USA.

Galeani, S., Menini, L., Tornambe, A., June 2003. A local observer for linearly observable nonlinear mechanical systems subject to impacts. In: Proceedings of the 2003 American Control Conference. Vol. 6. pp. 4760 - 4765 vol.6.

Goebel, R., Sanfelice, R., Teel, A., April 2009. Hybrid dynamical systems. Control Systems Magazine, IEEE 29 (2), 28-93.

Goebel, R., Sanfelice, R., Teel, A., 2012. Hybrid Dynamical Systems: modeling, stability, and robustness. Princeton University Press.

Goebel, R., Teel, A., 2006. Solutions to hybrid inclusions via set and graphical convergence with stability theory applications. Automatica 42 (4), 573 - 587.

Heemels, W., Brogliato, B., 2003. The complementarity class of hybrid dynamical systems. European Journal of Control 9 (2-3), 322-360.

Leine, R., van de Wouw, N., 2008. Stability and convergence of mechanical systems with unilateral constraints. Lecture Notes in Applied and Computational Mechanics. Springer Verlag.

Menini, L., Tornambè, A., November 2001a. Dynamic position feedback stabilization of multi-degrees-of-freedom linear mechanical systems subject to non-smooth impacts. IEE Proceedings of Control Theory and Applications 148 (6), 488-496.

Menini, L., Tornambè, A., 2001b. Velocity observers for linear mechanical systems subject to single non-smooth impacts. Systems \& Control Letters 43, 193-202.

Morarescu, I., Brogliato, B., 2010. Trajectory tracking control of multiconstraint complementarity Lagrangian systems. IEEE Transactions on Automatic Control 55 (6), 1300-1313.

Sanfelice, R., Goebel, R., Teel, A., 2007a. Invariance principles for hybrid systems with connections to detectability and asymptotic stability. IEEE Transactions on Automatic Control 52 (12), 2282-2297.

Sanfelice, R., Teel, A., Sepulchre, R., 2007b. A hybrid systems approach to trajectory tracking control for juggling systems. In: IEEE Conference on Decision and Control. New Orleans (LA), USA, pp. 5282-5287.

Teel, A., Forni, F., Zaccarian, L., 2011. Lyapunov-based sufficient conditions for exponential stability in hybrid systems. IEEE Trans. Aut. Cont., submitted. See also http://control.disp.uniroma2.it/zack/ftp/submitted/ ExpHybrid.pdf.

Tornambe, A., 1999. Modeling and control of impact in mechanical systems: Theory and experimental results. IEEE Trans. Aut. Cont. 44 (2), 294-309. 